\title{
ESCALAS ALOMÉTRICAS: UN JUSTO CRITERIO EN EL PROCESO DE EVALUACIÓN EN LAS CIENCIAS DEL MOVIMIENTO HUMANO
}

\author{
José Moncada Jiménez \\ Anthony Lachowetz
}

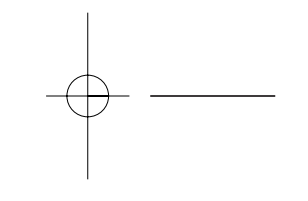

Resumen: El propósito del estudio fue comparar el tiempo de carrera en estudiantes de sexto grado de Costa Rica y los Estados Unidos de Norteamérica e investigar la influencia de la masa corporal sobre el tiempo de carrera. A los estudiantes $(N=274)$ se les midió la masa corporal y el tiempo en recorrer $1609 m$ a pie. Los análisis estadísticos incluyeron ANOVA factorial $2 \times 2$ (país x género) para las variables de masa corporal y tiempo de carrera; $y$ un análisis de regresión para crear un indice que ajustara los puntajes del tiempo de carrera y dejara de lado el efecto confusor de la masa corporal. Se obtuvo que los niños y las niñas costarricenses presentaron una menor masa corporal y corrieron más rápido los 1609 m que sus contrapartes estadounidenses $(p<0.05)$. En ambas muestras, los niños y las niñas que presentaban una mayor masa corporal tendian a ser penalizados con el método tradicional para asignar puntajes. Por ello, se calculó un indice de corrección, el cual ajustaba los tiempos de carrera tomando en cuenta las diferencias en la masa corporal. Se concluye que la técnica de las escalas alométricas es una berramienta justa que puede incorporarse en el proceso de evaluación en el área de las ciencias del movimiento bumano, y se recomienda la elaboración de normas que tomen en cuenta la influencia de la masa corporal de los participantes; así como la aplicación de las escalas alométricas a otras pruebas de la aptitud física.

\section{Palabras Clave}

Alometría, educación física, medición y evaluación, niños.

\section{Introducción}

En el área de las ciencias del movimiento humano han existido ciertas desventajas cuando se usan las normas con referencia de criterio para cuantificar los resultados de las pruebas de aptitud física. Primero, las normas con referencia de criterio pueden establecerse arbitrariamente, lo cual puede ser catastrófico si no se cuenta con la preparación académica o el consejo apropiado. De acuerdo con Pate y Hohn (1994), la forma en que se establecen las normas con referencia de criterio en el campo de la educación física es, en cierta medida, desconocida. Con excepción de la prueba de caminata y carrera de $1609 \mathrm{~m}$ (1 milla) de la batería de pruebas del FitnessGram (Cureton y Warren, 1990), el procedimiento exacto con el que se establecen los estándares de las pruebas no ha sido claramente revelado. A pesar de que siempre existe algún tipo de juicio para establecer puntajes de corte, es importante que los estándares se basen en sólidos criterios científicos. Segundo, la proporción de personas clasificadas correctamente en una medición de tipo criterio, basada en un estándar o 
puntaje mínimo es en sí misma, un reflejo de su validez. Hasta la fecha no se han reportado estudios comprensivos acerca de la validez de las pruebas de aptitud física con referencia de criterio, ni de los efectos de la clasificación incorrecta de los ejecutantes (Pate y Hohn, 1994). Finalmente, uno de los mayores problemas de las pruebas de aptitud física evaluadas con estándares establecidos con referencia de criterio, es que dichos estándares no son los mismos para pruebas idénticas de otras baterías de pruebas. Por ejemplo, en los Estados Unidos de Norteamérica, el puntaje del percentil 85 de la prueba de caminata y carrera de 1609 m (1 milla) para niños de 12 años de edad es la siguiente: a) Pruebas del FitnessGram = 10:00 min.; b) Pruebas del Physical Best $=9: 00$ min.; y c) Pruebas del President's Challenge $=7: 11 \mathrm{~min}$. Es evidente la discrepancia de 2 a $3 \mathrm{~min}$. que existe entre estas tres baterías de pruebas, aún tratándose de la misma prueba. Claramente se puede notar que los estándares que existen actualmente para un mismo elemento de la aptitud o salud física (i.e., resistencia cardiovascular) son diferentes.

Por otra parte, también existen algunas diferencias menores para otros componentes de la aptitud física, como por ejemplo la composición corporal, las pruebas de resistencia y de fuerza muscular (e.g., lagartijas, abdominales), y para las pruebas de flexibilidad. Las discrepancias en los estándares establecidos por los diferentes grupos hace reflexionar acerca de cuál es la base teórica para la elaboración de esos estándares y de su propia validez (Pate y Hohn, 1994).

Como se ha mencionado, ninguno de los diferentes grupos presentados ha descrito detalladamente cómo han desarrollado los estándares. Sin embargo, los modelos que se han usado para desarrollar los estándares si se conocen (e.g., para la caminata y carrera de $1609 \mathrm{~m}$ de Fitness Gram = empírica, normativa, juicio). El modelo usado para establecer otros tipos de estándares para pruebas de salud física para niños y jóvenes probablemente es similar al usado por FitnessGram; aunque el juicio de cómo deben establecerse los estándares ha sido obviamente distinto.

Discrepancias como éstas han llevado a otros investigadores (Jackson, 1986; Katch y Katch, 1974; Vandenburgh y Mahar, 1995; Vandenburgh, Mahar, y Chou, 1995), a estudiar qué tan apropiadas y válidas pueden ser las escalas alométricas (EA) en la medición y evaluación de actividades de fuerza y resistencia (e.g., pruebas de resistencia cardiovascular, pruebas de fuerza de manos) comúnmente utilizadas en las clases de educación física.

El uso de las EA se basa en relaciones matemáticas simples. En ellas, se asume que la relación verdadera entre el resultado de una prueba (e.g., correr y caminar 1609 m) y una variable antropométrica (e.g., masa o peso corporal, o masa libre de grasa) es curvilínea, pasa por el origen (sugiriendo que alguien con masa corporal $=0$ posee un tiempo en la prueba de $1609 \mathrm{~m}=0$ ), y que es de la forma: $y=b x^{a}$; en donde " $y$ " es el resultado en la prueba, " $x$ " es la variable a corregir (escala) o la variable antropométrica, " $b$ " es una constante multiplicativa, y " $a$ " es el exponente (Vandenburgh, Mahar, y Chou, 1995).

Para el presente trabajo, en el que se intentó comparar el tiempo (T) alcanzado por niños costarricenses y estadounidenses en la prueba de correr y caminar $1609 \mathrm{~m}$, y la masa o peso corporal (MC), la ecuación asumiría la siguiente forma: $T=b(M C)^{a}$. Si se divide $\mathrm{T}$ entre $\mathrm{MC}^{\mathrm{a}}$ se crea un nuevo índice del tiempo de carrera, el cual es igual a una constante, $b=T \cdot M C^{-a}$. La variable corregida, $T$. $M C^{-a}$, está, teóricamente, libre del efecto del peso de la persona, lo cual sugiere que la nueva variable ha sido ajustada o corregida para la masa corporal del estudiante. De este modo, se demuestra que el objetivo de las EA es el de crear una nueva variable en la que se puedan observar las diferencias entre los individuos dejando de lado (ajustando) el peso del participante. Al ajustar los puntajes, se estaría evaluando justamente, ya que en la práctica cotidiana del educador físico lo que se conoce es únicamente el peso total del 
estudiante (y a veces ni eso), pero no se sabe la proporción de grasa, músculo y hueso que componen ese peso. De esta manera, se podría tener en el campo a dos estudiantes de la misma edad, sexo, estatura, y con el mismo peso corporal total, y sin embargo, la composición del peso podría ser totalmente diferente.

Finalmente, los puntajes derivados de las EA pueden ser calculados fácilmente, aún por aquellas personas con una escasa preparación en análisis estadísticos. Lo simple de este proceso podría ayudar a comparar puntajes obtenidos (e.g., tiempos) entre estudiantes en las clases de educación física ajustando o dejando de lado el peso corporal. Para una detallada explicación acerca de las EA se recomienda revisar el artículo de Vandenburgh, Mahar, y Chou (1995).

Las carreras de distancia fija, en las que se mide el tiempo que una persona tarda en recorrer la distancia, se han usado ampliamente para estimar la condición cardiovascular o el consumo máximo de oxígeno $\left(\mathrm{VO}_{2 \text { máx }}\right)$, particularmente en pruebas de campo en las que la medición directa del $\mathrm{VO}_{2 \operatorname{máx}}$ no puede llevarse a cabo debido al elevado costo de los instrumentos de medición. Estos métodos se han usado tradicionalmente debido a la alta correlación entre las variables $\mathrm{VO}_{2 \text { máx }}$ (expresado por unidad de masa corporal) en $\mathrm{ml} \mathrm{O}_{2} \cdot \mathrm{kg}^{-1} \cdot \mathrm{min}^{-1}$, y el tiempo de carrera en una prueba de distancia fija (e.g., 1609 m). Sin embargo, personas con una mayor masa corporal (i.e., mayor peso) tienden a ser penalizadas en la evaluación si se usa esta manera de expresar los valores, debido a la desproporcionada variación en la relación entre el $\mathrm{VO}_{2 \text { máx }}$ y la MC. Como se mencionó anteriormente, la $\mathrm{MC}$ es una variable relativa que no brinda información acerca de la composición corporal del participante en términos de porcentaje de tejido graso, tejido libre de grasa, tejido muscular, o tejido óseo. Es por ello que una persona con mayor peso no necesariamente refleja a una persona con mayor cantidad de grasa corporal o menor desarrollo muscular.
De hecho, otros investigadores (Astrand y Rodahl, 1986; Berg, 1987) han sugerido que el $\mathrm{VO}_{2 \text { máx }}$ debería expresarse en $\mathrm{ml} \mathrm{O}_{2} \cdot \mathrm{kg}^{-}$ $0.7 \cdot \min ^{-1}$, de manera que se pudiese corregir la influencia de la masa corporal y se pudiesen llevar a cabo comparaciones entre sujetos. Es por ello que el propósito del estudio fue comparar el tiempo de carrera en la prueba de 1609 m en niños y niñas costarricenses y estadounidenses, y utilizar las escalas alométricas, primero, para corregir el tiempo de carrera de los niños y niñas, y segundo, para proponer desarrollar normas que tomen en cuenta la influencia de la masa corporal, las cuales teóricamente estarían libres del efecto confusor del peso de la persona.

\section{Metodología}

\section{Participantes}

Muestra Estadounidense. Una muestra de niños $(n=137)$ de sexto grado de la localidad de Framingham, Massachusetts, participaron voluntariamente en el estudio. La muestra consistió de 68 hombres y 69 mujeres. Cada participante presentó una carta de consentimiento informado firmada por su responsable legal, en la que daban por entendido el propósito del estudio y las mediciones que se llevarían a cabo.

Muestra Costarricense. Una muestra estratificada de niños de sexto grado de escuelas públicas y privadas fueron seleccionadas aleatoriamente, como parte de un estudio acerca de la salud física en los niveles escolares y colegiales (Fernández, Pearson, Moncada, Salas, González, y Picado, 1997). La muestra final de niños de sexto grado consistió de 982 participantes, de los cuales aleatoriamente se tomó una submuestra de 137 estudiantes ( $n=63$ niños, $n=74$ niñas) para compararla con la muestra estadounidense. Los padres o encargados legales de los niños firmaron un formulario de consentimiento de participación voluntaria en el estudio, el cual se recolectó el día en que se llevaron a cabo las mediciones. 


\section{Procedimientos}

La muestra costarricense (CR) utilizó la prueba de caminar y correr 1609 m del Physical Best, desarrollada por la Alianza Americana para la Salud, Educación Física, Recreación, y Danza (AAHPERD, por sus siglas en inglés) (1988). Por su parte, la muestra estadounidense (USA) utilizó la prueba de 1609 $\mathrm{m}$ del President's Challenge, desarrollada por el President's Council on Physical Fitness and Sports (1992). Aunque las pruebas han sido publicadas por diferentes instituciones, la validez de ambas es lógica y el protocolo en sí es idéntico.

La masa corporal (MC) en kilogramos fue medida tanto a los niños de la muestra CR como los de la muestra USA, en una balanza de plataforma marca Detecto®. Se usó un tallímetro para determinar la estatura de los participantes en centímetros. Luego de medir los $1609 \mathrm{~m}$ de distancia que componen la prueba de carrera, se determinó el número de vueltas al circuito que los participantes tenían que completar corriendo o caminando. El número de vueltas al perímetro establecido fue distinto en cada institución debido a que el espacio físico no era igual. Luego de un ligero calentamiento se llevó a cabo la prueba de $1609 \mathrm{~m}$. El tiempo (T) que cada participante tardó en correr o caminar el circuito se registró en minutos y segundos.

\section{Análisis Estadístico}

Para llevar a cabo el análisis de los datos se utilizó el Paquete Estadístico para la Ciencias Sociales (SPSS, por sus siglas en inglés) (1997). Se describieron las características de los participantes con medias aritméticas y desviaciones estándar $(\mathrm{M} \pm \mathrm{DS})$, y se realizaron análisis de varianza factoriales (ANOVA $2 \times 2$ ) por país y género para las variables dependientes masa corporal (MC) y tiempo de carrera (T).

Para desarrollar un índice que dejara de lado el peso de los participantes, se procedió a realizar un análisis de regresión lineal, el cual se justifica de la siguiente manera. Para la variable " $y$ ", la variable de corrección " $x$ ", y la función que mejor describe su relación, $y=b x^{a}$, la EA es un procedimiento de regresión lineal que resuelve el valor de " $a$ " (Vandenburgh y Mahar, 1995).

Para el caso de los tiempos de los niños de sexto grado, el valor $y=\mathrm{T}$ (min) y el valor $x=\mathrm{MC}(\mathrm{kg})$. Para que se pueda aplicar regresión a dicha función, ésta debe tomar una forma lineal. Por consiguiente, si se calcula el $\log _{10}$ a ambos lados de la ecuación, se transforma la relación en una de tipo lineal, resultando en la siguiente ecuación: $\log _{(\mathrm{y})}=$ (a) $\log _{(\mathrm{x})}+\log _{(\mathrm{b})}$. Los coeficientes de $\log _{(\mathrm{MC})} \mathrm{y}$ $\log _{(\mathrm{T})}$ son los valores correctos para una apropiada corrección del $\mathrm{T}$.

Finalmente, y debido a que uno de los supuestos teóricos más importantes en el análisis de regresión lineal se refiere a la normalidad en la distribución de los valores, se estudió, por medio de la prueba estadística de Kolmogorov-Smirnov, la normalidad de la distribución de los valores $\log _{(\mathrm{MC})} \mathrm{y} \log _{(\mathrm{T})}$ tanto para hombres como para mujeres, por país de procedencia.

Para el presente estudio, se aplicó análisis de regresión lineal a la variable dependiente (o criterio), $\log _{(\mathrm{T})}$, y a la variable independiente (o predictora), $\log _{(\mathrm{MC})}$. Los hombres y las mujeres no deberían combinarse dentro del mismo análisis de regresión lineal sin antes haber identificado que sus respectivos valores eran parecidos, cuando se analizaran por separado. Por ejemplo, si los valores de cada sexo fueran significativamente distintos, no tendría sentido tratar de combinar ambos grupos de puntajes para una comparación a posteriori. Sin embargo, si los valores de los hombres y las mujeres fueran similares, entonces sería apropiado combinar ambos grupos de puntajes y calcular otro análisis de regresión lineal para obtener diferentes multiplicadores " $b$ ", pero con idénticos valores " $a$ " (Nevill, Ramsbottom, y Williams, 1992). Los valores " $b$ " distintos reflejarían el efecto del sexo, mientras que los valores " $a$ " 
similares mostrarían el efecto de la masa corporal en el tiempo de carrera y caminata en la prueba de $1609 \mathrm{~m}$ para hombres y para mujeres (Vandenburgh y Mahar, 1995).

\section{Resultados}

En el estudio participaron 274 estudiantes de sexto grado. Las características de los participantes por país y por género se presentan en la Tabla 1. Del ANOVA 2 x 2 (país $\mathrm{x}$ género) para la variable dependiente MC se obtuvo una interacción estadísticamente significativa $(p=0.036)$. Al analizar los efectos principales se encontró que la interacción estaba explicada por el país de procedencia de los participantes $(p=0.000)$, con promedios de MC mayores en la muestra USA $(\mathrm{M}=49.62 \pm 13.77 \mathrm{~kg})$ comparada con la muestra CR $(\mathrm{M}=44.38 \pm 10.22)$; y no por el género de los estudiantes $(p>.05)$.

Por otra parte, con el ANOVA $2 \times 2$ (país x género) para la variable dependiente T se obtuvo diferencias significativas por país $(p=0.023)$ y por género $(p=0.000)$. Sin importar el género, los costarricenses obtuvieron un promedio de tiempos significativamente menores $(\mathrm{M}=10.33 \pm 1.88 \mathrm{~min}$. $)$ que el de los estadounidenses $(\mathrm{M}=10.81 \pm 2.37$ min.) en la prueba de caminata y carrera de $1609 \mathrm{~m}$. Por su parte, y sin importar el país de procedencia, los hombres corrieron significativamente más rápido que las mujeres $(\mathrm{M}=9.82 \pm 2.05$ min. vs. $\mathrm{M}=11.27 \pm 1.99$ min., respectivamente).

Tabla I

Estadística descriptiva* de los escolares de sexto grado $(\mathrm{N}=274)$

\begin{tabular}{|c|c|c|c|}
\hline País & Sexo & $\begin{array}{c}\text { Masa Corporal } \\
(\mathrm{kg})\end{array}$ & $\begin{array}{c}\text { Tiempo de Carrera } \\
\text { (min) }\end{array}$ \\
\hline Costa Rica & $\begin{array}{l}\text { Hombres }(n=63) \\
\text { Mujeres }(n=74)\end{array}$ & $\begin{array}{l}43.29 \pm 10.46 \\
45.31 \pm 9.99\end{array}$ & $\begin{array}{l}9.38 \pm 1.70 \\
11.15 \pm 1.63\end{array}$ \\
\hline Estados Unidos & $\begin{array}{l}\text { Hombres }(n=69) \\
\text { Mujeres }(n=68)\end{array}$ & $\begin{array}{l}51.67 \pm 16.38 \\
47.54 \pm 10.17\end{array}$ & $\begin{array}{l}10.23 \pm 2.27 \\
11.41 \pm 2.33\end{array}$ \\
\hline
\end{tabular}

* media aritmética \pm desviación estándar.

Los tiempos obtenidos en la carrera de 1609 m fueron estudiados para determinar si se tendía a penalizar a los niños con mayor masa corporal. El coeficiente de correlación producto momento de Pearson entre la MC y el T para la muestra CR fue significativamente diferente de cero $(r=0.49$; $p=0.000)$. La misma situación ocurrió en la muestra USA, en la que se obtuvo $r=0.22$ $(p=0.008)$. En otras palabras, tanto para la muestra de CR como para la muestra de USA, el tiempo de carrera aumentó significativamente conforme aumentaba la masa corporal del participante, lo cual quiere decir que los niños con mayor masa corporal tendían a ser penalizados si se les evaluaba por medio de la asignación de puntajes convencionales. 
Para analizar la normalidad en la distribución de los puntajes transformados $\log _{(\mathrm{MC})}$ y $\log _{(\mathrm{T})}$ tanto para hombres como para mujeres, por país de procedencia, se utilizó la prueba de Kolmogorov-Smirnov (KS). Para la muestra CR se obtuvo $K S \log _{(\mathrm{MC})}=0.51 ; p=$ 0.961 ; $\mathrm{yS} \log (\mathrm{T})=0.49 ; p=0.969$, lo cual indicó normalidad en las distribuciones. Para la muestra USA también se determinó normalidad en las distribuciones, con $K S \log _{(\mathrm{MC})}=$ $0.56 ; p=0.914 ;$ y $K S \log { }_{(\mathrm{T})}=0.78 ; p=0.582$.

Con base en los resultados obtenidos con la prueba $K S$ se procedió a aplicar el procedimiento de EA para cada país. Se encontró que los exponentes de la MC fueron $a=$ 0.438 y $a=0.220$ para CR y USA, respectivamente. Estos exponentes indicaron que si se deseaba corregir el efecto de la masa corporal sobre el tiempo de carrera, los participantes de la muestra CR deberían dividir el tiempo de carrera entre la masa corporal elevada al exponente $a=0.438$ (i.e., T/MC $\mathrm{MC}^{0.438}$ ); mientras que los participantes de la muestra USA deberían dividir el tiempo de carrera entre la masa corporal elevada al exponente $a=$ 0.220 (i.e., $\mathrm{T} / \mathrm{MC}^{0.220}$ ).

De este modo, una vez obtenido cada exponente, se debía correlacionar la masa corporal con el nuevo índice obtenido, de manera que se pudiese verificar la validez de la nueva variable. Para la muestra CR, se obtuvo $r=-0.08$, lo cual sugiere que no existe relación entre el tiempo de carrera y la masa corporal del participante (después de ajustar por diferencias en MC). De manera similar, la muestra USA obtuvo un $r=-0.03$.

\section{Discusión}

En este estudio se compararon los tiempos en una carrera de 1609 en niños y niñas costarricenses y estadounidenses, y se utilizó el procedimiento de las EA para estudiar la influencia del peso corporal en el tiempo de carrera. Los resultados del estudio indicaron que los niños y niñas con mayor peso corporal, pero no necesariamente obesos, tendían a ser penalizados si se les aplicaba el método tradicional de evaluación por medio de normas. Si bien es cierto que se necesita algún tipo de estándar para evaluar grandes cantidades de estudiantes, como es común en las clases de educación física, en realidad no se ha hecho algún intento para controlar el efecto confusor del peso corporal sobre los tiempos de carrera de los estudiantes. El no controlar de alguna manera el efecto confusor de la masa corporal, podría perjudicar en la evaluación a los estudiantes con mayor desarrollo muscular de la misma manera en que perjudicaría a los estudiantes obesos.

La gran variación en el tamaño y el peso corporal de los estudiantes de sexto grado garantiza algún tipo de investigación acerca de la validez y la utilidad de los estándares que se usan actualmente para evaluar a los niños y niñas; todo ello con el propósito de brindar una evaluación más justa sin importar el peso corporal. Por consiguiente, el uso de estándares con referencia de criterios que tomen en cuenta el peso corporal parecen ser una lógica alternativa en lugar de los estándares tradicionales.

En la Tabla 2 se puede observar el efecto relativo de los dos métodos usados para determinar el puntaje que recibiría un estudiante de sexto grado en la prueba de $1609 \mathrm{~m}$. Ya que tiempos de carrera más bajos se consideran mejores, los puntajes de la Tabla 2 tienen que interpretarse de tal forma; es decir, números menores en la columna de tiempo indican que los estudiantes corrieron más rápido. En la muestra USA, los estudiantes que obtuvieron puntajes relativamente moderados, de acuerdo al rango de percentil, (e.g., sujeto \# $204=\mathrm{RP}_{50}$ ) aparentemente aumentaron su puntaje con la escala alométrica (i.e., sujeto $\# 204=R P_{70}$ ). Sin embargo, los estudiantes que obtuvieron puntajes en los rangos bajos de la muestra (e.g., sujeto \# $206=\mathrm{RP}_{10}$ ), obtuvieron cambios mejores en la clasificación alométrica (e.g., sujeto \# $206=\mathrm{RP}_{30}$ ).

Por supuesto, no todo estudiante se beneficia del método de clasificación alométrica. 
Por ejemplo, en la muestra de niños costarricenses, el estudiante \# 66 obtuvo una clasificación en el rango de percentil $90\left(\mathrm{PR}_{90}\right)$; sin embargo, cuando se ajustó su tiempo tomando en cuenta su masa corporal, éste mismo niño fue clasificado en el $\mathrm{PR}_{60}$, posiblemente porque ya presentaba una masa corporal ba- ja (i.e., un niño muy delgado). A pesar de ello, las EA parecen ser justas para clasificar objetiva y justamente a aquellos estudiantes que presentan un peso corporal alto, el cual no necesariamente indica una mayor cantidad de masa grasa (es decir, podría tratarse de un niño muy musculoso).

Tabla 2

Comparación de dos métodos para evaluar el rendimiento en una prueba de carrera de 1609 m y su rango de percentil (RP) correspondiente en una selección de niños de sexto grado de Costa Rica y los Estados Unidos de Norteamérica.

\begin{tabular}{|c|c|c|c|c|c|c|}
\hline País & $\begin{array}{c}\text { Estudiante } \\
\#\end{array}$ & $\begin{array}{l}\text { Peso } \\
(\mathrm{kg})\end{array}$ & $\begin{array}{l}\text { Tiempo en } 1609 \mathrm{~m} \\
\text { (min:seg) }\end{array}$ & $\mathrm{RP}_{\text {tradicional }}$ & Índice & $\mathrm{RP}_{\text {nuevo }}$ \\
\hline Costa Rica & $\begin{array}{c}136 \\
62 \\
61 \\
66\end{array}$ & $\begin{array}{l}75.23 \\
71.70 \\
66.82 \\
30.00\end{array}$ & $\begin{array}{r}15: 13 \\
8: 95 \\
11: 58 \\
8: 90\end{array}$ & $\begin{array}{l}10 \\
60 \\
20 \\
90\end{array}$ & $\begin{array}{l}2.28 \\
1.38 \\
1.84 \\
2.01\end{array}$ & $\begin{array}{l}30 \\
90 \\
50 \\
60\end{array}$ \\
\hline USA & $\begin{array}{l}206 \\
204 \\
274 \\
203\end{array}$ & $\begin{array}{c}127.27 \\
89.09 \\
74.09 \\
73.64\end{array}$ & $\begin{array}{l}14: 50 \\
10: 00 \\
12: 07 \\
11: 00\end{array}$ & $\begin{array}{l}10 \\
50 \\
40 \\
30\end{array}$ & $\begin{array}{l}4.99 \\
3.72 \\
4.68 \\
4.27\end{array}$ & $\begin{array}{l}30 \\
70 \\
60 \\
40\end{array}$ \\
\hline
\end{tabular}

En resumen, como era de esperarse, aquellos niños que presentaban una mayor masa corporal tendían a ser penalizados con el método tradicional de asignación de puntajes; es decir, cuando no se tomaba en cuenta la masa corporal del niño. Lo anterior es válido sin importar el país de procedencia ni el género del estudiante. Se observa claramente que cuando sí se toma en cuenta el peso del participante, se puede asignar puntajes más justos, lo cual, desde todo punto de vista, vuelve el proceso de medición y evaluación más objetivo, especialmente en el área de las ciencias del movimiento humano, en donde en una parte importante del tiempo de clase se realizan pruebas de campo que sustituyen las costosas y sofisticadas pruebas de laboratorio.

En este estudio, los niños estadounidenses presentaron un peso promedio mayor que el de sus contrapartes costarricenses; sin embargo, éstos últimos corrieron más rápido. Si bien se sabe que el somatotipo de ambas muestras es distinto, se observó claramente una relación inversa entre la masa corporal y el tiempo para correr los 1609 m. Se podría argumentar que los niños costarricenses son más activos físicamente que los niños estadounidenses, lo cual es beneficioso para su salud en general, lo cual se vio reflejado en mejores tiempos de carrera.

Futuras investigaciones deberían estudiar la utilidad de aplicar EA a otros componentes de la aptitud física (e.g., lagartijas, abdominales) y elaborar nuevas normas. Para ello, las autoridades educativas deberían establecer una agenda y escuchar los criterios técnicos de los profesionales de las ciencias del movimiento humano. En el caso de Costa 
Rica, se ha hecho un intento de establecer normas nacionales (Fernández et al., 1997); las cuales han sido publicadas parcialmente; sin embargo, se requiere de mayor voluntad política para sufragar los costos inherentes a un proyecto de tal magnitud.

\section{Referencias bibliográficas}

American Alliance for Health, Physical Education, Recreation, and Dance. Physical Best. Reston, VA: AAHPERD, 1988.

American College of Sports Medicine. ACSM's guidelines for exercise testing and prescription (5th ed.). Media, PA: Williams \& Wilkins, 1995.

Astrand, P. O., \& Rodhal, K. Textbook of work physiology. New York, NY: McGrawHill, 1986.

Berg, U. The influence of body mass in crosscountry skiing. Medicine and Science in Sports and Exercise, 19, 324-331, 1987.

Cureton, K. J., \& Warren, G. W. Criterion-referenced standards for youth health-related fitness tests: A tutorial. Research Quarterly for Exercise and Sport, 61(1), 7-19, 1990.

Fernández, A., Pearson, G., Moncada, J., Salas, R., González, C., y Picado, M. Informe Nacional: Evaluación de la salud física de estudiantes de la educación general básica. San José, Costa Rica: Ministerio de Educación Pública, Universidad de Costa Rica, Escuela de Medicina UCR, Programa para el Mejoramiento de la Calidad de la Educación - PROMECE, Convenio MEP-UCR (IIMEC), 1997.

Jackson, A. Strength measurement: Controlling for individual differences. Journal of Physical Education, Recreation, and Dance, 57, 82-84, 1986.
Gobierno de Costa Rica. La Gaceta (8-95). Decreto Ejecutivo \# 23893. San José, Costa Rica: Gobierno de Costa Rica, 1995.

Katch, V. L., \& Katch, F. I. Use of weight-adjusted oxygen uptake scores that avoid spurious correlations. Research Quarterly, 45, 447-451, 1974.

Nevill, A. M., Ramsbottom, R., \& Williams, C. Scaling physiological measurements for individuals of different body size. European Journal of Applied Physiology, 65, 110-117, 1992.

Pate, R. R., \& Hohn, R. C. Health and fitness through physical education. Champaign, IL: Human Kinetics, 1994.

SPSS, Inc. SPSS Base System ${ }^{T M}$ 7.5. Chicago, IL: SPSS Inc., 1997.

Vandenburgh, P. M., \& Mahar, M. T. Scaling of 2-mile run times by body weight and fat-free weight in college-age men. Journal of Strength and Conditioning Research, 9(2), 67-70, 1995.

Vandenburgh, P. M., Mahar, M. T., \& Chou, C. H. Allometric scaling of grip strength by body mass in college-age men and women. Research Quarterly for Exercise and Sport, 56(1), 80-84, 1995.

\section{Nota}

Parte de los datos presentados en este estudio son propiedad del Ministerio de Educación Pública de Costa Rica (MEP), del Instituto de Investigación para el Mejoramiento de la Educación Costarricense (IIMEC), y del Laboratorio de Rendimiento Físico y Salud (LARENFISA) de la Escuela de Medicina de la Universidad de Costa Rica (UCR). 